\title{
Experiencia pragmática de medios y entornos digitales
}

\author{
Romualdo Gondomar Miñana \\ Elisava. Escuela Universitaria de Diseño e Ingeniería de Barcelona \\ rgondomar@elisava.net \\ https://orcid.org/0000-0003-1670-6182
}

Pragmatic experience of digital media and environments

\begin{abstract}
RESUMEN
Las experiencias cotidianas se nutren del cúmulo de información que nos proporciona nuestro entorno y utilizamos esos datos para construir nuestro conocimiento y orientar

nuestras actividades. De forma similar, para orientar la comprensión de los entornos digitalizados, las experiencias demandan componentes significativos que orienten y faciliten la comunicación interactiva. El mundo digital demanda, quizás más que el físico, la participación de todos los órganos sensoriales $y$, por ello, parece oportuno ampliar la concepción de las actividades que desplegamos en el mundo tecnológico. Una perspectiva que valore todos los componentes que participan en una experiencia digital y aporte una concepción holística que integre: a) cómo participan todos los sentidos en la captación de esta nueva realidad, b) qué instrumentos y recursos utilizan los medios que transmiten información y, c) como todo ello se interrelaciona con el sustrato social y cultural de los individuos. Por esta razón, se precisan recursos teóricos e instrumentos metodológicos que faciliten la comprensión de los mecanismos de comunicación que vehiculan las interacciones entre los individuos y los elementos presentes y configuradores de la realidad digital. Fruto de la revisión de diferentes aportaciones al estudio de la interacción de los entornos digitalizados, se plantea que una concepción pragmática de las experiencias en espacios digitalizados puede contribuir a delimitar las dimensiones comunicativas que participan y guían nuestras actividades. Por ello, se propone un posicionamiento que facilite la delimitación de los elementos que son relevantes en los procesos de interacción y el reconocimiento de los diferentes registros de comunicación que guían y orientan nuestras actividades en función del contexto.
\end{abstract}

\section{PALABRAS CLAVE}

Medios digitales, Comunicación interactiva, Experiencia, Pragmática, Metodología

\section{ABSTRACT}

Everyday experiences are nourished by the accumulation of information that our environment provides us and we use these data to build our knowledge and guide our activities. Further, to guide the understanding of digitized environments, experiences demand significant components that guide and facilitate interactive communication. The digital world demands, perhaps more than physical, the participation of all sensory organs and, therefore, it seems appropriate to broaden the conception of the activities we deploy in the technological world. A perspective that values all the components that participate in a digital experience and contributes a holistic conception that integrates: a) how all the senses participate in capturing this new reality, $b$ ) what instruments and resources use the media that transmit information and, c) how all this is interrelated with the social and cultural substrate of individuals. For this reason, theoretical resources and methodological instruments are needed to facilitate the understanding of communication mechanisms that convey the interactions between individuals and the elements present and configurators of digital reality. The outcome of the review of different contributions to the study of the interaction of digitized environments, it is proposed that a pragmatic conception of experiences in digitized spaces can help to delimit the communicative dimensions that participate and guide our activities. Therefore, a pragmatic positioning is proposed for facilitate the delimitation of the elements that are relevant in the interaction processes and the recognition of the different communication registers that guide and direct our activities according to the context.

\section{KEYWORDS}

Digital media, Interactive communication, Experience, Pragmatics, Methodology 


\section{Introducción}

Los avances tecnológicos de los medios y dispositivos digitales están dirigiendo la configuración de las relaciones interactivas que se proponen a las personas. Los nuevos aparatos electrónicos amplían los tradicionales registros de comunicación y extienden sus ámbitos de intervención a entornos cada vez más próximos e inmediatos. Los sistemas de comunicación que se implantan para intercambiar información con los usuarios alteran el desarrollo habitual de nuestras actividades y requieren procesos de aprendizaje y de memorización. Y, además, la simultaneidad y convergencia de procesos comunicativos en las experiencias digitales determina la trascendencia significativa de nuestras actividades. Una nueva dimensión de la experiencia que demanda una concepción teórica que considere la importancia de la producción del significado como impulsora de la adecuación, la trasformación y la construcción de nuestra dimensión social (Couldry y Hepp, 2017).

El presente ensayo trata de ofrecer una respuesta a esta demanda: un posicionamiento teórico que facilite instrumentos metodológicos y analíticos para abordar la complejidad que se presenta en la experiencia digital de los medios interactivos de comunicación. Con este fin, y con la intención de localizar los componentes que intervienen en la interacción comunicativa se revisan, en primer lugar, algunos de los estudios más relevantes sobre la distinción y confluencia de diferentes dimensiones comunicativas en nuestro entorno. A continuación, se analizan los ámbitos de comunicación del espacio interactivo a partir del nuevo paradigma conversacional que promueven los nuevos dispositivos digitales y los elementos que intervienen en las experiencias de los individuos. Para, finalmente, examinar cómo se configuran las experiencias digitales y que elementos son determinantes en el contexto de la situación.

Las principales aportaciones que derivan de los ámbitos investigados nos permiten aplicar un enfoque pragmático de la experiencia comunicativa y establecer conexiones con las actividades interactivas que se promueven desde el entorno digital. En las aportaciones finales destacan las relaciones entre el posicionamiento teórico propuesto y las características pragmáticas de los componentes relevantes en los procesos de interacción.

\section{La diversidad del entorno comunicativo}

La pluralidad de medios de comunicación presentes en nuestro entorno cotidiano nos ofrece un conjunto de información tan diversa que determinan la necesidad de conocer como cada medio estructura la variedad de mensajes que nos trasmite. El conocimiento de las características de cada uno de esos registros de comunicación nos puede facilitar, en función de la situación y de acuerdo con nuestros objetivos, la gestión del valor significativo de los mensajes que recibimos y, así, podremos evaluar su influencia en los procesos de decisión que hemos de tomar.

En la última década, el análisis y clasificación de los diferentes registros comunicativos se ha abordado desde posiciones teóricas y disciplinares diversas. Cada una de esas perspectivas de estudio ha distinguido y clasificado los distintos niveles de información que usamos habitualmente en nuestras actividades cotidianas. Entre ellas, destacamos la realizada por Echeverría (1999) que adopta una concepción contextual de las relaciones comunicativas, participantes principales de la construcción social de la realidad, para clasificar los vínculos de convivencia que la actividad humana establece en los espacios inmediatos de interacción. Centrando su atención en las características particulares generadas por la revolución tecnológica propuso una distinción sistemática de los diversos escenarios sociales por los que transitan las personas: un entorno natural, el entorno urbano o artificial y un "nuevo" entorno tecnológico o digital.

Desde la perspectiva teleológica que adopta el autor, los individuos intervienen directamente en su entorno recurriendo a procesos cognitivos que les permiten asimilar, adaptar y transformar sus escenarios en función de sus necesidades y de las relaciones de convivencia que conllevan las actividades humanas. Los diferentes ámbitos comunicativos con los que interactuamos se intersectan y participan, simultáneamente, en la configuración de nuestras convenciones e instituciones socioculturales $\mathrm{y}$, aunque adopten formas representativas $\mathrm{y}$ formales diversas, no podemos considerarlas, ni valorarlas de forma aislada. La comprensión del alcance representativo del entorno habitual debe extenderse, por tanto, a la interpretación de un medio multidimensional en el que los individuos realizan sus experiencias. Por esta razón, adquiere importancia entender cómo se conceptualizan los diversos contenidos y la forma de representarse y exteriorizarse en función de un contexto determinado. Conocer los escenarios y dimensiones donde los sujetos infieren el conjunto de significados que emplean para interrelacionarse social y culturalmente. Así, podremos determinar el papel que los contenidos juegan en los procesos previos a la toma de decisiones de las personas y comprender como participan en la realización de sus actividades.

Si centramos nuestra atención en el ámbito de la transformación, en las relaciones que establecemos con los componentes utilizados para modificar el medio, podremos distinguir las principales dimensiones de la interacción cotidiana. El carácter somático y sensorial de las relaciones tangibles que los individuos establecen con su entorno próximo determina la naturaleza material de la primera dimensión, el entorno natural: espacio en el que se manifiestan claramente las capacidades perceptivas y motoras humanas. Por ello, en esta magnitud física, las posibilidades comunicativas y las limi- 
taciones interactivas están condicionadas, principalmente, por los estímulos que los sentidos son capaces de captar, por el significado atribuido a la información recibida y por la respuesta funcional ${ }^{1}$ que conduce nuestras competencias emocionales y sociales (Goleman, 1996). Un proceso de interrelación con el medio guiado por el significado que ofrecen los recursos inmediatos.

La segunda dimensión de Echeverría se corresponde con el carácter político del espacio artificial y urbano. El hecho de compartir las estructuras que emergen de la órbita física precedente, de socializarlas a través de los diversos medios e instrumentos culturales, determina como se manifiestan las actividades comunes. En esta dimensión política adquiere importancia y transcendencia el significado sociocultural de las instituciones compartidas y convencionalizadas. Las directrices que orientan y delimitan las actividades en este entorno de relaciones construidas se rigen por su adecuación social o por la manera de actuar común. En este ámbito, los procesos de interacción se articulan a partir de los diversos sistemas de comunicación que las personas han codificado como instrumentos para transmitir y ejercer sus prácticas habituales. La eficacia de estas mediaciones comunicativas radica en las oportunidades que ofrecen a los individuos para relacionarse entre sí, para compartir concepciones socioculturales y para establecer los lazos que configuran la comunidad.

Tanto la dimensión natural como la artificial procuran interrelaciones comunicativas e interactivas que se estructuran a partir de dos componentes presentes en todas las situaciones que experimentamos: el espacio y el tiempo. Sin embargo, en el entorno digital o tecnológico, la tercera dimensión que enumera Echeverría, estos dos elementos se implican de manera diferente en el devenir de los hechos. El tiempo y el espacio ven alterado su alcance y su proyección y ya no se limitan a concretar las condiciones habituales. Estos dos factores, al combinarse de forma distinta, generan un ámbito vivencial en el que convergen, simultáneamente, diversas dimensiones comunicativas.

La revolución digital de los escenarios de interacción, sobreponiéndose a las limitaciones naturales de los seres humanos, transforma el sistema de relación espaciotemporal preestablecido. Se nos ofrecen formas novedosas de participación que alternan diferentes dimensiones comunicativas. El tránsito sincrónico por un amplio conjunto de contenidos se extiende de forma reticular, facilitando que se singularicen contextos digitalizados que se diferencian de los entornos precedentes. La conciliación tecnológica del espacio y el tiempo que impulsan los medios digitalizados facilita que la sociedad de la información y del conocimiento proponga nuevos escenarios de interacción.

La comprensión de los medios comunicativos que manejan las tecnologías digitales solicita atender a una nueva realidad que sigue estando participada por los procesos interactivos surgidos del entorno natural y social. En la dimensión digital adquiere una especial relevancia y significación cómo la ordenación de los contenidos, en función del espacio y el tiempo, determina el sentido de la secuencia de interrelación comunicativa. La configuración del proceso interactivo digital debe seguir contemplando como intervienen los sistemas sensoriales y psicológicos de las personas y coordinarlos con las limitaciones y las capacidades tecnológicas que nos ofrecen los nuevos medios de comunicación digitalizados.

Scolari (2008) propuso el término hipermediaciones para definir los procesos que se generan en este nuevo espacio comunicativo, donde las diversas posibilidades de interacción que nos brinda el entorno digital se caracterizan por la confluencia de diferentes dimensiones comunicativas. Gracias a las nuevas oportunidades tecnológicas, la pluralidad de sujetos, medios y lenguajes que concurren en un mismo momento del espacio y del tiempo, los propios procesos interactivos posibles, surgen de "la confluencia de lenguajes, la reconfiguración de los géneros y la aparición de nuevos sistemas semióticos caracterizados por la interactividad y las estructuras reticulares" (2008, p. 115). Desde este posicionamiento las actividades humanas no constituyen fenómenos estancos y regulados sino que cambian y evolucionan gracias al conjunto de decisiones que, externalizándose, responden sincrónicamente a los diferentes sistemas significativos que convergen en una misma unidad espaciotemporal.

En la práctica real, el ser humano transita por los diferentes entornos de manera simultánea y continua. Va captando y seleccionando la información transmitida y articulando sus decisiones a partir de un espacio conceptual común que es conocido y compartido socioculturalmente y que está integrado en el seno de cada colectividad. Nuestras experiencias cotidianas nos aportan el conjunto de datos y de información que precisamos para elaborar nuestro conocimiento vivencial y nos permiten reconocer el significado contextual que guía y orienta nuestras actividades. La aplicación del espacio conceptual común y compartido facilita las conexiones pragmáticas necesarias para afrontar las situaciones que experimentamos. Smith (1996) asimila este proceso cognitivo experimental, que facilita la comprensión de las nuevas experiencias, a una inducción inmanente ${ }^{2}$. En este procedimiento, la participación de los individuos es esencial para dar continuidad a los conocimientos adquiridos en los diferentes entornos, para aplicar el sentido inherente resultante de las prácticas.

En el entorno digital el sistema se estructura a través de la práctica que posibilita identificar las características de los medios tecnológicos, su relevancia comunicativa y como trascienden a nuestras acciones y decisiones. Para conectar y comprender los mensajes y condiciones que ahora nos proponen los nuevos medios McLuhan los relacionó expresamente con los recursos precedentes, como una forma eficaz para entender aquello que nos muestran, ofrecen y sus efectos: "a 
practical means of perceiving the action and effects of ordinary human tools and services" (1988, p. 98). Esta perspectiva comprende la concepción pragmática de la experiencia comunicativa y permite centrar la atención en el valor semiósico que generan las acciones de las personas, conectando nuestro conocimiento y nuestra interpretación de la realidad.

\section{La expansión de las experiencias digitales}

El ambiente interactivo digital se concibe como un espacio en el que se generan, se transmiten y se perciben los diversos contenidos comunicativos que emiten los artefactos creados por los seres humanos. Un contexto tecnológico, real o virtual, contenedor de una pluralidad de dimensiones y productor de diferentes registros comunicativos que se expanden de forma reticular en el espacio y en el tiempo y donde confluyen espacios conceptuales diversos. Una concepción que podemos adoptar para entender la complejidad que se presenta al abordar su estudio y análisis. Messaris y Humphreys (2006) distinguen las características que permiten comprender cómo los medios digitales están transformando la comunicación humana. Unas propiedades que están relacionadas naturalmente con la percepción y los procesos cognitivos utilizados en el tratamiento de la información. Los autores, dirigen su atención a aspectos que intervienen directamente en la configuración de los medios de comunicación, desde su conceptualización hasta su producción, y centran su estudio en el uso y comprensión que del medio realizan las personas. Para ellos, la evolución digital de los medios se basa en la recreación de las relaciones que se generan en el mundo exterior. Una revolución que se apoya, por un lado, en la capacidad que brindan los dispositivos tecnológicos para reproducir estímulos que nos excitan sensorialmente (simulación de imágenes, de sonidos y voz y de sensaciones táctiles) y, por otro, por producir y emitir contenidos significativos relacionados con las acciones y actividades humanas (captación de expresiones faciales, movimientos oculares, entonaciones de voz, etc.).

Recientemente, Rose (2014) y Greengard (2015), también centran su atención en las relaciones comunicativas que los sujetos establecen con los entornos tecnológicos a partir de los sentidos y de las habilidades físicas. De acuerdo con ambos estudiosos, esta es la mejor opción para que los seres humanos comprendan las posibilidades que les muestran los dispositivos digitales. Consideran que debemos diseñar elementos e interfaces tangibles que faciliten la interacción comunicativa con las personas. Impulsado y promoviendo las interrelaciones sensitivas y motoras los individuos podrán usar los instrumentos de forma adecuada y establecer conexiones emocionales con el entorno digital, empleando gestos, expresiones y movimientos naturales.

En paralelo, la comunicación con instrumentos, medios y escenarios digitales se está transformando gracias a las posibilidades que ofrece la conectividad. El desarrollo tecno- lógico facilita la interconexión entre los diversos entornos y el acceso a todo tipo de información que se procesa en función de unas determinadas reglas y criterios que orientan su contextualización y permiten reconocer la realidad inmediata. El considerado creador del concepto de Internet de las cosas $(\mathrm{IoT})^{3}$, Kevin Ashton, reflexionó sobre el alcance de los objetos conectados a la red relacionándolo con las capacidades y limitaciones humanas:

We're physical, and so is our environment. Our economy, society and survival aren't based on ideas or information - they're based on things. You can't eat bits, burn them to stay warm or put them in your gas tank. Ideas and information are important, but things matter much more (2009).

La aparición de aparatos y artefactos inteligentes en nuestros entornos vuelve a cuestionar la manera de interrelacionarnos y de dialogar con unos objetos que "piensan", que son capaces de adaptarse, "inteligentemente", a las circunstancias cambiantes del entorno gracias a la información que usan. Un proceso de captación de la realidad que facilita la adecuación de los objetos a las condiciones del momento y, en función de los referentes estructurados y acumulados que poseen, la capacidad de responder en consonancia a aquello que se les demanda. El tratamiento tecnológico de datos que se realiza trasciende los métodos tradicionales de procesamiento de la información. La adquisición progresiva de interacción de los dispositivos digitalizados, que amplía y actualiza sus funciones habituales, deriva de la información que manejan y de los procesos de ordenación que utilizan para simular el razonamiento humano.

La configuración de los objetos con nuevas prestaciones interactivas debe sustentar y orientar la interacción digital de nuestras experiencias, adaptándose a un nuevo paradigma basado en información para dialogar. Shati (2016), por ejemplo, enumera cuales son las principales fuentes de información que manejan los robots para ejecutar actividades estructuradas y tomar decisiones en concordancia a los patrones que tienen preestablecidos: la visión (análisis visual de las imágenes capturadas, reconocimiento de elementos del entorno e identificación de las emociones humanas); el movimiento (la detección, comprensión y significado del movimiento y la correspondiente sincronización de las respuestas); los sonidos (atender y escuchar los diferentes niveles de sonidos que se generan en el espacio para entender y seguir las conversaciones humanas, comprender contextualmente aquello que se dice y detectar las emociones que contienen). El conjunto de dimensiones comunicativas enumeradas facilita y vehicula nuestra conexión con los aparatos "inteligentes" y están supeditadas a los mecanismos que relacionamos con las emociones y la creatividad humana. Las diversas facetas comunicativas deben corresponderse con la capacidad de los objetos para entablar relaciones dialógicas con los suje- 
tos. Por ello, debemos superar los tradicionales sistemas de interacción comunicativa de estructuras jerarquizadas para implantar procedimientos reticulares que permitan a estos dispositivos reconocer e identificar las emociones e intenciones de las personas.

La principal diferencia entre los procesos de comunicación actuales, a través de artefactos inteligentes, y la comunicación automatizada con máquinas, precedente, se encuentra en el potencial emotivo que son capaces de trasmitir estos nuevos aparatos y su capacidad para empatizar con los usuarios. La incorporación de datos relacionados directamente con el comportamiento y emociones humanas está impulsando el desarrollo de nuevas realidades digitales como, por ejemplo, el campo de los videojuegos y el de la realidad virtual. En ambos mundos se amplían los canales de información, incorporando la interacción física mediante la simulación del movimiento corporal y se articulan los medios de comunicación a partir del concepto de presencia y de las particulares experiencias espaciotemporales de los individuos. Una concepción inmersiva que ubica la propia actividad en un momento concreto y determina la sensación humana de estar en un lugar, constituyendo y configurando la propia experiencia, y facilitando la estructuración de los procesos mentales de los individuos (Steuer, 1995).

\section{Contextualización de las situaciones digitales}

En las precedentes transformaciones del mundo artificial surgieron problemas relacionados con la aceptación y adaptación de los individuos a las novedades que se proponían. Cuestiones que ponían en crisis cómo los sujetos comprendían la incorporación de los avances tecnológicos y que demandaron revisar los procesos de adecuación a las prácticas cotidianas para acomodarlos a las expectativas de las personas. La resolución de estos dilemas, que corroboran las dependencias intrínsecas del propio proceso tecnológico, se ha abordado desde ópticas contextuales que han tratado de integrar todos los elementos participantes de los procesos interactivos digitales.

La confluencia de diversas dimensiones comunicativas, coincidentes en un mismo espacio temporal, constituye el punto de partida que adopta Dourish (2004) para sistematizar los procesos de comunicación y ordenar el flujo de información que se ofrece a los individuos. Para dotar de sentido al proceso interactivo se debe realizar un tratamiento de la información que concuerde con las capacidades de percepción, atención y reconocimiento de los individuos y relacionarse, directamente, con las capacidades y limitaciones que determinan el uso de los dispositivos digitales en cada situación. El autor concentra su atención en la comprensión de los flujos de datos que generan los dispositivos interactivos incorporando los preceptos principales de la teoría psicológica de la percepción formulada por Gibson (1986), en la que una asignación adecuada del valor de las relaciones significativas con las cosas se erige como uno de los factores clave en el desarrollo de gran parte de las actividades humanas (Roda, 2014). El conocimiento de las relaciones que se establecen entre elementos y las conexiones espaciotemporales que establecemos con los objetos nos permiten construir y actuar en nuestra realidad.

Los seres humanos actuamos intencionalmente para conseguir unos resultados y establecemos conexiones con todos los componentes del medio circundante al igual que cualquier otro sistema dedicado a realizar unas tareas específicas. Por tanto, también en nuestra comunicación con los medios digitales se genera una corriente continua de información que ordena y orienta la producción continua de acciones. Realizamos conexiones entre realidades, internas y externas, que activan los procesos de razonamiento necesarios para estructurar, ordenar y asimilar cada nueva situación. Identificamos y definimos la situación que directamente experimentamos gracias al tratamiento de la información que captamos de nuestro entorno mediante los diferentes canales sensoriales. El intercambio de información entre los medios y las personas permite identificar como participan los elementos contextuales en las decisiones que toman los individuos en el transcurso de sus actividades. De esta manera, las secuencias que ejecutamos, ordenadas y estructuradas por etapas, están determinadas por la información que nos llega a partir de unos "periféricos de entrada", nuestros órganos sensoriales, de unos dispositivos de "almacenamiento de datos", nuestra memoria, y de unos "periféricos de salida", nuestro sistema motor.

La comprensión de las situaciones se estructura a partir del conocimiento del mundo tal como lo percibimos, del conjunto de elementos que componen el entorno y de la ordenación de los procesos que realizamos. Como individuos, nos encontramos ante un sistema de correlaciones entre el entorno percibido y un contexto de acción. Dos sistemas distintos que conectamos mediante nuestros recursos cognitivos para que nos ayuden a tomar las decisiones más adecuadas. Un proceso comprensivo que genera respuestas dirigidas hacia la aceptación de la realidad en que estamos inmersos, que procuran una adecuación de los objetivos y que, finalmente, definen nuestro comportamiento. Intervenimos en nuestra realidad de modo intencional: "el comportamiento se adapta a finalidades $y$, en consecuencia, es artificial y revela solamente aquellas características del sistema de comportamiento que limitan la adaptación" (Castells, 2009, p. 97). Las actividades humanas se encaminan a conseguir unos determinados propósitos $y$, por tanto, cada acción que abordamos tiene un objetivo más o menos prefijado o una finalidad preestablecida ${ }^{4}$.

En términos sistemáticos, si seguimos a $\mathrm{H}$. Simon, la efectividad del proceso interactivo de comunicación se consigue a medida que se adquiere y almacena información sobre las situaciones, relacionando el mundo exterior con nuestras actividades, previendo los resultados de las interacciones y 
planificando los procesos comunicativos pertinentes para guiar las actividades de los individuos: "La capacidad de conseguir los objetivos depende de la construcción de asociaciones, que pueden ser simples o muy complejas, entre cambios específicos en los estados del mundo y las acciones específicas que (de forma predecible o no) los producen" (Simon, 2006, p. $146)^{5}$. En términos generales, relacionamos los diversos sucesos del entorno y la interpretación de la situación es la que guía y adapta nuestras respuestas al contexto en que actuamos.

En las situaciones que normalmente experimentamos debemos relacionar los significados que asignamos al conjunto de cualidades ambientales percibidas con nuestra toma de decisiones, seleccionando aquellas características que identificamos como impulsoras de nuestras acciones. El estudio y análisis de las cualidades percibidas es un tema recurrente en los diferentes enfoques de estudio de los medios digitales. Básicamente, porqué facilita la comprensión de como los individuos perciben la información de su entorno, le atribuyen significado y valoran los elementos contextuales de comunicación y de interacción para alcanzar sus objetivos. Es decir, se traspasa la concepción real de la actividad a la propuesta interactiva para procurar una comunicación efectiva y productiva. Los comunicadores deben conocer, por tanto, cuales son los signos que intervienen en el proceso interactivo, cuales son los contenidos que pueden atribuirse a estos signos, los significados que las personas proyectan sobre estos contenidos y, por último, cómo determinan o guían la acción de las personas. Para Gee: "Primero, podemos verlo directamente en términos de contenido, es decir, qué signos tienen y cómo están organizados. En segundo lugar, podemos verlo en términos de cómo las personas interactúan con ese contenido o entre ellos sobre ese contenido" (2004, p. 73). La significación de las cualidades percibidas se fundamenta, por lo tanto, en la oferta representativa que trasmiten los componentes de interacción. Una representatividad que, como información formalizada expresamente, debe orientarse para facilitar la comprensibilidad de la situación. La direccionalidad representativa, claramente intencional, usa los diversos medios disponibles para sistematizar el proceso comunicativo que participa directamente en la toma de decisiones de la experiencia digital. Esta relación nos dota de razones suficientes para afirmar que la asignación de significados depende de las asociaciones que conectan el mundo exterior con nuestras acciones, del reconocimiento de cómo nuestros actos inciden y alteran el medio y, en definitiva, de la adquisición y consolidación de conocimientos a través de las experiencias de interacción. Por lo tanto, gracias a nuestra exposición a nuevas experiencias podremos adquirir información que nos ayude a construir y/o corroborar nuestros criterios de toma de decisiones y adecuar nuestras actividades a los acontecimientos de la realidad.

Exteriorizamos y proyectamos nuestras capacidades de obrar buscando un equilibrio entre las condiciones contextuales y nuestro conocimiento. Siguiendo a Castells (2009), nuestra concepción de la realidad no es preferentemente objetiva o subjetiva, sino un resultado del proceso cognitivo que mezcla, comparando y complementando, lo que sucede en el mundo real con nuestra experiencia acumulada. Son las conductas que realizan los individuos en el espacio las que moldean las prácticas sociales y las transforman. Si atendemos a la forma cómo se realizan estas interacciones podremos identificar cuáles son los patrones o repeticiones que definen identidades típicas y podremos normalizarlos y emplearlos:

We learn not only the basic communicative signs, but also patterns of how to communicate: the way to 'question', to 'answer', to 'discuss', etc., is based on certain social patterns - 'rules' based in institutional facts - which we learn during our socialization (Couldry y Hepp, 2017, p. 30).

Todo un conjunto de significados que podemos localizar tanto en las relaciones intersubjetivas que se establecen socialmente entre personas como en la interpretación de los signos disponibles en el espacio de intervención. Por tanto, la significación, derivada de la representatividad conceptual, puede utilizarse para construir espacios compartidos y planificar estructuras de interacción acordes con el uso y finalidades que expectativas y necesidades comunes puedan determinar. Los contenidos se trasmiten a partir de una representación y significación cultural compartida que promueve la diversidad y variabilidad de las experiencias humanas. Desde esta perspectiva, podemos recurrir al concepto de espacios de afinidad de Gee (2005) que nos aporta una concepción contextual que fomenta la comprensión mutua de significados. Precisamente en el esfuerzo de como concebir y ofrecer a otros individuos una configuración compartida del espacio es donde el autor hace recaer la importancia significativa de los espacios afines. La concepción intersubjetiva del contexto, organizada alrededor de los espacios comunes de todos los individuos que forman una sociedad o una cultura determinada, es también compartida por Miller $(2012,2017)$ y Lahlou (2012) para quiénes, además, la interacción genera relaciones en sentido inverso: la cultura da forma y complementa la información que captamos a través de nuestros sentidos (Grice, 1991). Se hace extensiva, así, una perspectiva analítica del proceso comunicativo de la interacción que se basa en el conocimiento e interpretación de las actividades compartidas y socializadas.

La interpretación cultural de los contenidos y de los signos que nos comunica el espacio también incide, directamente, en nuestra manera de interpretar la información sensorial que percibimos a nuestro alrededor. En cada una de nuestras experiencias nos orientamos por una significación que comparamos con nuestro conocimiento de experiencias pasadas, con aquellos modelos que hemos acumulado con el paso del tiempo. Una providencia que conecta directamente con la tradición pragmatista de una experiencia participada por el conocimiento acumulado. 


\section{La concepción pragmática de la experiencia digital}

La concepción pragmática de la experiencia nos permite rastrear cómo se asigna sentido a los elementos conformadores del contexto y nos proporciona una descripción útil de los medios que nos facilita entender una situación y tomar nuestras decisiones. La localización de las raíces pragmáticas de los referentes comunicativos surge de ópticas analíticas que tratan de ampliar el conocimiento sobre la organización de las relaciones del entorno digital a partir de la experiencia propia como fuente de significación, de los procesos convencionalizados de elaboración y representación de la información y de la selección y tratamiento de unos medios que facilitan la emisión y la trasmisión de mensajes de forma eficaz para hacerlos accesibles a la comunidad. Es importante, por tanto, contextualizar desde un primer momento la interacción comunicativa, ya que las características pertinentes de la propia situación se erigen como uno de los elementos principales para delimitar el significado de los canales de comunicación utilizados y el alcance de los diversos mensajes que se trasmiten en cada una de las secuencias de la actividad.

El pragmatismo va más allá de una concepción de la naturaleza intrínseca de las cosas y de sus características relacionales y dirige sus objetivos epistemológicos hacia la planificación de actividades significativas (Dewey, 2000; Rorty, 1996). En nuestras experiencias enlazamos y relacionamos el conjunto de actos comunicativos que se producen para poder interpretar y entender las situaciones interactivas que vivimos. Estas conexiones generan secuencias narrativas que se ordenan en función de una lógica discursiva que ordena el espacio y el tiempo dotando de coherencia y de unidad experimental al conjunto de actos que realizamos en los procesos de interacción.

En el ámbito digital es manifiesta la necesidad de planificar las secuencias de interacción comunicativa para ofrecer a los usuarios unos referentes conceptuales que faciliten la ordenación de sus actos. La competencia comunicativa radica, por tanto, en relacionar el ordenamiento conceptual con la interacción del proceso que se propone. El uso de secuencias de comunicación compartidas y convencionalizadas favorece que los destinatarios reconozcan cada situación. La cohesión comunicativa de la unidad situacional se logra a partir de la intencionalidad pragmática que asigna significados a la sucesión de actos comunicativos y los regulariza para que sean extensibles a toda la comunidad. Desde un posicionamiento pragmático podemos concebir los procesos de interacción como una unidad operativa (Pericot, 1987) y entender la experiencia como una posibilidad continua de acción en la que participan las pautas socializadas (Searle, 1997; Tomasello, 2013).
Los objetivos de la comunicación se dirigen a ayudar y/o compartir información con los demás de algún aspecto y de alguna manera. Para Davidson, esta acción intencional de la comunicación: "Encuentra su fundamento en la teoría del significado y de la interpretación y estos dos componentes determinan el carácter público y común de las relaciones comunicativas" (1992, p. 35). La teoría pragmática del significado, que se basa en los principios establecidos por Grice (1991), analiza los procesos de comunicación a partir de la inferencia y de la relevancia de los contenidos reconocidos, compartidos y valorados por la colectividad. Se otorga, como también hacia Morris (1985), una naturaleza social a la actividad comunicativa que implica tener en cuenta también las condiciones y los elementos que participan necesariamente en el comportamiento colectivo del espacio común, condición indispensable para el intercambio de información que determina todo proceso comunicativo (Tomasello, 2013). Para la tradición pragmática solo los medios que por su relevancia se incorporen a los hábitos socioculturales entraran a formar parte del sistema común de significados (Lewis, 1956; Goodman, 1995; Dewey, 2000; Mead, 2008).

Los conceptos que utilizamos en los procesos de comunicación están modelados por las relaciones sociales que se basan en la significación inferida del conjunto de experiencias de los individuos. En concordancia al posicionamiento epistemológico que adopta Lewis (1956), podemos afirmar que en nuestros intercambios comunicativos relacionamos los términos a partir de unas definiciones comunes que utilizamos como pautas de conceptualización y, al compartir una misma idea del mundo, se facilita el proceso de comunicación. La comprensión situacional no se agota en ella misma sino que vehicula la socialización de esa misma actividad. El recurso que genera la conceptualización es la propia interacción social que se sobrepone a las diversidades y particularidades de las experiencias subjetivas y que determina la construcción y la configuración de la colectividad mediatizada (Couldry y Hepp, 2017). En este sentido, será la experiencia común la que origina y nutre la conceptualización pragmática gracias a la participación de todos los individuos que comparten conductas y cooperan para concretar, acordar y generalizar las convenciones que sustentan la sociedad.

De esta manera, serán las similitudes descubiertas en nuestras experiencias las que alimenten el valor intersubjetivo de los conceptos, recolectando los elementos comunes que contribuyen a la estabilización y relativa permanencia de los mismos. Es así cómo se construye y se participa en el sistema conceptual que utilizamos para relacionarnos cotidianamente y que se erige como un esquema, de carácter pragmático, que organiza nuestras actividades. En la realidad del ámbito digital los significados también surgen de la interacción entre las personas y los medios, el mismo intercambio comunicativo implica interacción (Moragas, 2011). Instrumentalizamos los medios para formalizar mensajes que dirigen el proceso de 
mediación entre aquello que se trasmite y el comportamiento de las personas, es decir, para facilitar que los individuos se apropien y utilicen el contenido significativo del contexto interactivo para obrar en función de sus objetivos.

Específicamente, el tratamiento metodológico de la experiencia pragmática centra su atención en las relaciones entre el entorno percibido y el conocimiento inferido a través de la acción. El objetivo consiste en facilitar la comprensión del proceso semiósico que progresivamente va aportando razones para que los individuos puedan lidiar con las situaciones a que se enfrentan habitualmente. La distinción de los diferentes niveles de comunicación que originan los procesos inferenciales facilita la exploración del significado de los medios en función de las situaciones concretas y de las relaciones contextuales que se pueden establecer. Estamos inmersos en un contexto cargado de contenidos socioculturales de los que inferimos los significados que nos ayudan a ordenar nuestras experiencias. Ahí radica la importancia del significado contextualizado (Givón, 1989). Una interrelación que dirige las intenciones comunicativas y la selección de los datos más relevantes del contexto donde se producen los acontecimientos. En este sentido, debemos tener presente que la trasmisión ostensiva y concreta de significados o mensajes se basa en el intercambio de datos que genera el propio acto comunicativo que permite relacionar los efectos que produce el significado inferencial (Sperber y Wilson, 1994).

Las metodologías pragmáticas orientan nuestra atención al contexto común de significados que generan las actividades que llevamos a cabo y que configuran nuestra conducta. El análisis, comprensión e interrelación de la experiencia comunicativa trata de identificar y distinguir las diversas dimensiones significativas que intervienen en la comprensión de nuestras experiencias. Por tanto, nos proponen procesos analíticos que utilizan como elementos de trabajo los contenidos inferidos directamente a través de nuestras prácticas cotidianas. Unos contenidos que nos ayudan a posicionarnos en nuestra interpretación de la situación, a relacionarnos con los medios que captan nuestra atención y a valorar unos significados concretos (Gondomar, 2014, 2016). Este proceso de valoración, basado en la interpretación útil de los significados fruto de nuestros procesos de interacción, relación y selección, orienta y dirige el sistema de toma de decisiones que rige nuestro comportamiento ${ }^{6}$.

\section{Conclusiones}

Los puntos en común que presentan la diversidad de experiencias digitales que nos ofrecen los avances tecnológicos, y la aproximación a sus distintos marcos de estudio realizada, nos permite afirmar que las dimensiones comunicativas de los entornos digitalizados se estructuran a partir de la configuración contextual que da soporte a la exposición de la propia actividad. Nuestras actividades nos ofrecen un conjunto de datos que usamos para dar sentido y ordenar nuestras experiencias $\mathrm{y}$, consecuentemente, utilizamos los significados inferidos como elementos que nos orientan hacia unos objetivos o finalidades concretos. Podemos concebir, así, un proceso de interacción digital participado, directa y efectivamente, por los individuos que guía sus experiencias como respuesta a todo aquello que en el entorno posee significado. Un sistema de relación estructurado por la capacidad representativa de los medios utilizados para planificar el proceso de comunicación. Hemos podido examinar como las circunstancias situacionales muestran unas características pragmáticas que se articulan a partir del uso relacional de la significación de las experiencias.

La contextualización de los procesos comunicativos que se suceden en el entorno digital nos ha permitido ubicar como participan los medios en la configuración de los sistemas interactivos. La identificación de estructuras pragmáticas ha puesto de relieve como las conexiones conceptuales que realizan las personas guían su participación en los procesos interactivos. Un posicionamiento teleológico que ubica directamente la experiencia digital en el contexto real en el que se generan los procesos de intercomunicación y que nos facilita delimitar la pertinencia de las relaciones relevantes de las secuencias comunicativas. En este sentido, la intencionalidad pragmática del espacio digitalizado se erige como uno de los elementos determinantes para planificar la trasmisión de significados concretos. Los procesos que nos facilitan la interrelación con el espacio nos facultan recursos que nutren nuestro sistema comunicativo y conceptual, sistemas en los que se apoyan los procesos de razonamiento que conducen nuestras actividades.

Si atendemos a las dimensiones comunicativas que dotan de contenido a las experiencias podemos centrar nuestra atención en el sentido real de los actos que se producen, en la utilidad que nos proporcionan. Así es como estaremos dando valor al sentido que nos ofrecen los recursos digitales como instrumentos orientadores de la acción. Saber cómo se estructura el proceso experimental de adquisición del significado y como se emite a través de los medios tecnológicos contribuye a dotar de contenidos y articular aquello que queremos comunicar.

$Y$, finalmente, consideramos conveniente adoptar una concepción participativa del propio proceso de experimentación digital. El concepto de espacios afines adoptado por las tecnologías digitales emana, en nuestra opinión, directamente de las pautas pragmáticas de interpretación que recogen qué aspectos de la experiencia son relevantes para la comunidad y como pueden utilizarse para facilitar la sociabilidad. Una perspectiva teórica que integra las dimensiones particulares, compartidas y comunes de nuestras propias experiencias. 


\section{Notas al final de página}

1. Una de las principales aportaciones realizadas por Goleman consiste en conectar nuestras actividades prácticas con los procesos de cognición articulados por nuestras emociones socializadas.

2. El sentido inmanente de los signos, constitutivo del pragmatismo, se relaciona más con los posibilidades de hacer que de pensar y pretende equilibrar la interpretación conceptual de nuestras experiencias.

3. (IoT) Internet of things.

4. Dewey (1950) define las "end-in-views", finalidades a la vista o en perspectiva, como determinantes para la comprensión de la unidad situacional.

5. Herbert Simon, Nobel de Economía por sus estudios sobre la racionalización de la toma de decisiones, es uno de los principales referentes en el ámbito de la inteligencia artificial.

6. Un esquema analítico que debería complementarse con recursos proporcionados por la etnometodología y la sociología de la interacción y relacionarse con los participantes del proceso a través de conceptos ergonómicos y de las propuestas desarrolladas por la psicología cognitiva.

\section{Referencias}

Ashton, K. (2009). That 'Internet of Things' Thing. RFID Journal, 22. Recuperado de http://www.rfidjournal.com/article/view/4986, consultado el 15/02/18

Castells, M. (2009). Comunicación y poder. Madrid: Alianza.

Couldry, N., y Hepp, A. (2017). The mediated construction of reality. Malden, MA: Polity Press.

Davidson, D. (1992). Mente, mundo y acción: claves para una interpretación. Bellaterra: I.C.E. de la Universidad Autónoma de Barcelona.

Dewey, J. (1950). Lógica: teoría de la investigación. Buenos Aires: Fondo de Cultura Económica.

Dewey, J. (2000). La Miseria de la epistemología: Ensayos de pragmatismo. Madrid: Biblioteca Nueva.

Dourish, P. (2004). Where the Action is: The Foundations of Embodied Interaction. Cambridge, Mass.: MIT Press.

Echeverría, J. (1999). Los señores del aire: Telépolis y el tercer entorno. Barcelona: Destino.

Gee, J. P. (2004). Situated language and learning: a critique of traditional schooling. London [etc.]: Routledge.

Gee, J. P. (2005). Semiotic Social Spaces and Affinity Spaces: From the Age of Mythology to Today's Schools. En D. Barton, y K. Tusting (eds.) Beyond communities of practice: Language, power and social context (pp. 214-232). Cambridge: Cambridge University Press.

Gibson, J. J. (1986). The Ecological approach to visual perception. New York: Psychology Press.

Givón, T. (1989). Mind, code, and context: Essays in pragmatics. Hillsdale (N.J.) [etc.]: Lawrence Erlbaum Associates.

Goleman, D. (1996). Inteligencia emocional. Barcelona: Kairós.

Gondomar, R. (2014). Aportacions metodològiques al disseny de les coses des del pragmatisme. Comunicació: Revista de Recerca i d'Anàlisi, 31(1), 89-110.

Gondomar, R. (2016). El Llenguatge de la utilitat: una anàlisi pragmàtica de les coses. El disseny com a planificador de les relacions comunicatives entre persones i coses (Tesis doctoral). Universitat Pompeu Fabra. Barcelona. Recuperado de http://hdl.handle. net/10803/385734

Goodman, N. (1995). De la mente y otras materias. Madrid: Visor.

Greengard, S. (2015). The internet of things. Cambridge, Mass.: MIT Press.

Grice, H. P. (1991). Studies in the way of words. Cambridge, Mass.: Harvard University Press.

Lahlou, S. (2012). Designing user-friendly augmented work environments: From meeting rooms to digital collaborative spaces. London: Springer.

Lewis, C. I. (1956). Mind and the world-order: outline of a theory of knowledge. New York: Dover.

McLuhan, M., y McLuhan, E. (1988). Laws of media: the new science. Toronto: University of Toronto Press.

Mead, G. H. (2008). La Filosofía del presente. Madrid: Boletín Oficial del Estado.

Messaris, P., y Humphreys, R. L. (eds.) (2006). Digital media: transformations in human communication. New York: Peter Lang.

Miller, K. (2012). Playing along: Digital games, YouTube, and virtual performance. Oxford: Oxford University Press.

Miller, K. (2017). Playable bodies: dance games and intimate media. New York, NY: Oxford University Press.

Moragas, M. (2011). Interpretar la comunicación: estudios sobre medios en América y Europa. Barcelona: Gedisa.

Morris, C. W. (1985). Fundamentos de la teoría de los signos. Barcelona: Paidós.

Pericot, J. (1987). Servirse de la imagen: un análisis pragmático de la imagen. Barcelona: Ariel.

Roda, C. (2014). Human Attention in Digital Environments. Cambridge: Cambridge University Press.

Rorty, R. (1996). Consecuencias del pragmatismo. Madrid: Tecnos.

Rose, D. (2014). Enchanted objects: Design, human desire, and the Internet of things. New York: Scribner.

Sathi, A. (2016). Cognitive (internet of) things: Collaboration to optimize action. New York, NY: Palgrave Macmillan.

Scolari, C. (2008). Hipermediaciones: elementos para una teoría de la comunicación digital interactiva. Barcelona: Gedisa.

Searle, J. R. (1997). La Construcción de la realidad social. Barcelona: Paidós.

Simon, H. A. (2006). Las Ciencias de lo artificial. Granada: Comares.

Smith, B. C. (1996). On the origin of objects. Cambridge (Mass.): MIT Press.

Sperber, D., y Wilson, D. (1994). La Relevancia: Comunicación y procesos cognitivos. Madrid: Visor.

Steuer, J. (1995). Defining Virtual Reality: Dimensions Determining Telepresence. En F. Biocca, y M. Levy (eds.), Communication in the age of virtual reality (pp. 33 -56). Hillsdale, N.J.: L. Erlbaum Associates.

Tomasello, M. (2013). Los orígenes de la comunicación humana. Buenos Aires: Katz. 
CV

Romualdo Gondomar Miñana. Doctor en Comunicación Social por la Universidad Pompeu Fabra de Barcelona con la tesis "El lenguaje de la utilidad: un análisis pragmático de las cosas" (ECL). Graduado en Diseño de producto por ELISAVA, Escola Universitària de Disseny i Enginyeria, UPF; BA (Hons) in Design, University of Southampton, UK; Graduado Superior en Diseño por la Escola d'Arts i Oficis "Llotja" de Barcelona. Investigador en Comunicación y Diseño. Docente del Grado en Diseño y del Grado en Ingeniería en diseño industrial de ELISAVA, UPF. Profesor de Metodología de la investigación en el Máster Universitario en Diseño y Comunicación de ELISAVA, UPF. Docente del Grado en Diseño y del Posgrado de Diseño de experiencia de usuario (UX) de la UOC. Docente del Postgrado en Investigación y Gestión del Diseño del ISEC, Lisboa.

Observatorio de Cibermedios

\section{https://observatoriocibermedios.upf.edu/}

\begin{tabular}{|l|l} 
upf. & $\begin{array}{l}\text { Universitat } \\
\text { Pompeu Fabra } \\
\text { Barcelona }\end{array}$ \\
\hline
\end{tabular}

Departamento de Comunicación

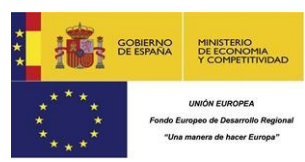

El Observatorio de Cibermedios es una producción del Grupo de Investigación en Documentación Digital y Comunicación Interactiva (DigiDoc) del Departamento de Comunicación de la Universitat Pompeu Fabra.

El Observatorio de Cibermedios (OCM) forma parte del proyecto del Plan Nacional "Creación y contenido interactivo en la comunicación de información audiovisual: audiencias, diseño, sistemas y formatos". CS02015-64955-C4-2-R (MINECO/ FEDER), Ministerio de Economía y Competitividad (España). 